\title{
Neurofibroma of the Cheek and Nasal Dorsum: A Case Report and a Mini Review
}

\author{
${ }^{1}$ Neelima Gupta, ${ }^{2}$ Ramandeep Kaur, ${ }^{3}$ Ashish Rai, ${ }^{4}$ Neelam Wadhwa, ${ }^{5}$ PP Singh
}

\begin{abstract}
Neurofibroma is a benign neoplasm derived from peripheral nerves. Most of these are associated with neurofibromatosis but may also occur as solitary lesions. When found in the neck, they are often located in the soft tissues. They are slow-growing masses, often asymptomatic and have been found most commonly in the oral cavity. We report a case of neurofibroma involving two separate and unusual sites of face, which are subcutaneous tissues of the cheek and dorsum of nose, and present a brief review of literature.
\end{abstract}

Keywords: Cheek, Neurofibroma, Nose, Peripheral nerve sheath tumor, Solitary.

How to cite this article: Gupta N, Kaur R, Rai A, Wadhwa N, Singh PP. Neurofibroma of the Cheek and Nasal Dorsum: A Case Report and a Mini Review. Int J Otorhinolaryngol Clin 2016;8(2):62-64.

Source of support: Nil

Conflict of interest: None

\section{INTRODUCTION}

Neurogenic tumors are relatively common tumors of the nervous system, but they form a very small percentage of all neoplastic lesions of the head and neck region. They include neurofibroma, schwannoma and neuroma, ganglioneuromas, ganglioneuroblastoma, and neuroblastoma. ${ }^{1}$ Schwannoma is the most common solitary neurogenic tumor followed by neurofibroma. ${ }^{1}$ Neurofibromas are circumscribed benign but uncapsulated tumors. The plexiform neurofibroma and multiple localized neurofibromas are characteristic of neurofibromatosis type 1 (NF1). ${ }^{1}$ They can arise from Schwann

\footnotetext{
${ }^{1,4}$ Associate Professor, ${ }^{2}$ Senior Resident, ${ }^{3}$ Assistant Professor ${ }^{5}$ Professor and Head

1,2,5 Department of Otorhinolaryngology, Guru Teg Bahadur Hospital and University College of Medical Sciences, New Delhi, India

${ }^{3}$ Department of Burns and Plastic Surgery, RML Hospital, New Delhi, India

${ }^{4}$ Department of Pathology, Guru Teg Bahadur Hospital and University College of Medical Sciences, New Delhi, India

Corresponding Author: Ramandeep Kaur, Senior Resident Department of Otorhinolaryngology, Guru Teg Bahadur Hospital and University College of Medical Sciences, New Delhi, India Phone: +919654323623, e-mail: raman_rdk41@yahoo.com
}

cells, neurons, fibroblasts, and perineural cells. ${ }^{2}$ Malignant transformation has been reported to occur in 2 to $16 \%$ of cases. ${ }^{3}$ They usually present as a slow-growing mass and can mimic any benign growth in the head and neck. The treatment of extracranial head and neck neurofibromas is mainly surgical, and the approach depends on the location and extent of tumor and the nerve involved.

As neurofibromas are commonly associated with NF1 and NF2, solitary involvement is rare. There are case reports describing solitary lesions, but very few studies have mentioned multiple sites involving simultaneously on face. This case report describes a case of neurofibroma, presenting as a nodular mass in the mid-right cheek and dorsum of nose. The lesion was approached through skin and completely resected followed by the reconstruction of defect using forehead and local advancement flap.

\section{CASE REPORT}

A 65-year-old man presented with the chief complaint of painless swelling over the right cheek and nose. He noticed the swelling about 6 months back which had shown a progressive increase in size. There was no history of trauma or previous surgery. He gave no history of paresthesia or tingling over the cheek. On examination, localized mobile nontender firm and oval swellings of about $4 \times 5 \mathrm{~cm}$ and $3 \times 4 \mathrm{~cm}$ were evident over the right cheek and dorsum of nose respectively (Fig. 1). The overlying skin appeared stretched and was partially adhered to the swelling. There was no loss of sensation over the cheek. All the cranial nerve examination was normal except second nerve that could not be tested because of bilateral corneal opacity. Intraoral and dental examinations were normal. The cervical lymph nodes were not palpable. The overall clinical presentation was suggestive of a benign subcutaneous lesion and differential diagnosis included lipoma, fibroma, leiomyoma, neuroma, and adenoma.

Fine needle aspiration of the swelling was reported as a benign peripheral nerve sheath tumor. The patient underwent complete excision of the tumor, and the defect was repaired using a forehead and local flap (Fig. 2), forehead flap based on supratrochlear artery covered nasal defect, and cheek defect repaired using Mustarde's local rotation advancement flap. Histopathological examination confirmed the diagnosis of a neurofibroma (Fig. 3). 


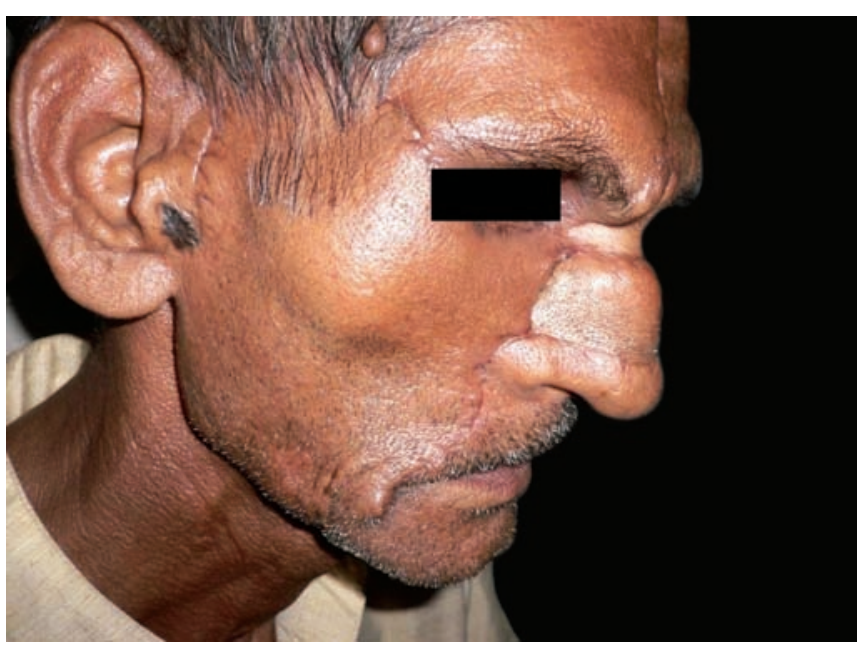

Fig. 1: Preoperative photograph of the lesion

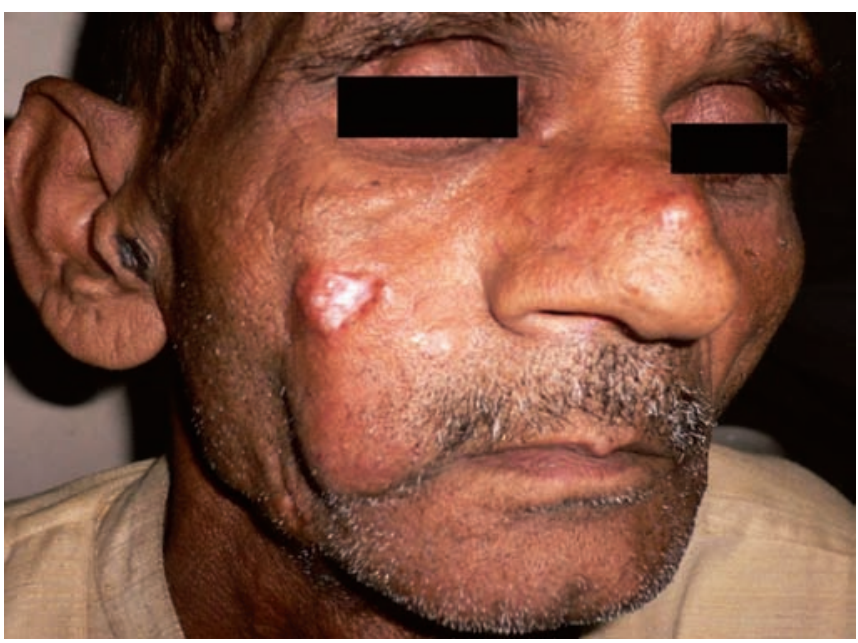

Fig. 3: Histopathological photograph showing spindle-shaped cells with wavy nuclei and pointed ends dispersed in loose connective tissue background (hematoxylin and eosin 200x) and inset showing positive reaction seen as brown colored pigment in several tumor cells, S-100 immunostain (400×)

The skin covered the soft tissue pieces that measured $9 \times 3.8 \times 2 \mathrm{~cm}$ and $2.5 \times 1.8 \times 0.5 \mathrm{~cm}$ respectively. Cut surface of both revealed gray white tumor with glistening surface. Sections showed a benign mesenchymal tumor composed of spindle-shaped cells dispersed loosely in an edematous and myxoid stroma. The nuclei were thin and wavy. There was no evidence of necrosis or mitoses. A variable number of tumor cells expressed S-100, a marker for schwannian differentiation. The case was diagnosed as a diffuse neurofibroma. Retrospective examination of the patient showed no skin lesions (café au lait macules) and no eye involvement. After a year, postoperative follow-up showed a good cosmetic outcome with no recurrence (Fig. 4).

\section{DISCUSSION}

Extracranial neurogenic tumors of the head and neck are relatively rare tumors, arising from the neural crest. ${ }^{1}$

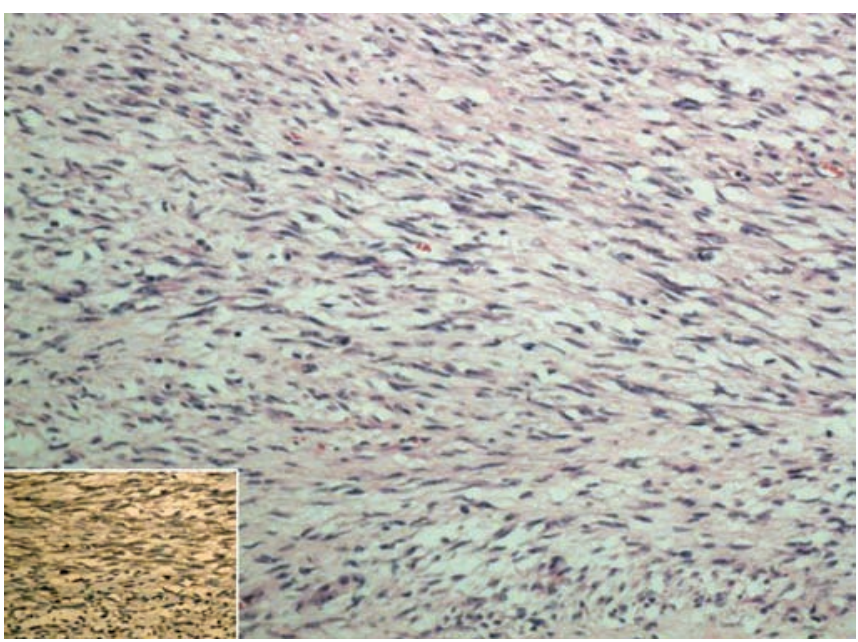

Fig. 2: Intraoperative photograph of the flap markings

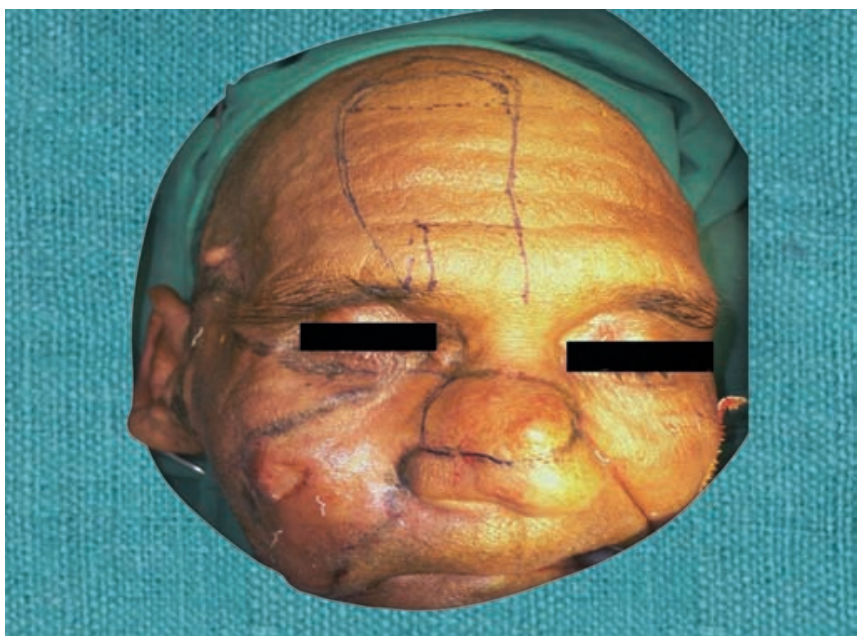

Fig. 4: Postoperative photograph of the patient

Schwann cells give rise to schwannoma and neurofibroma. ${ }^{1}$ Schwannoma is the most common followed by neurofibroma. ${ }^{1}$ Neurofibromas constitute about $25 \%$ of all neurogenic tumors in the head and neck and are often associated with von Recklinghausen's disease if they are multiple, but they need not be multiple and may exist as discrete entities. ${ }^{2}$ They show autosomal dominant pattern occurring either due to spontaneous mutation or from the passing of infected gene with complete penetrance and variable expression. ${ }^{4}$ They are well-differentiated nonencapsulated tumors and are composed of Schwann cells, perineural cells, and fibroblasts and arise within the endoneurium. ${ }^{1,3}$ The nerve fibers are incorporated within the tumor; therefore, nerve fibers cannot be separated and resection may sacrifice the nerve of origin. They are rarely malignant. ${ }^{3}$ The aetiology behind solitary neurofibroma is still unknown. Marocchio et $\mathrm{al}^{2}$ considered solitary neurofibroma to be hamartomatous malformation rather than neoplastic.

Majority of patients are asymptomatic and present with a mass in the neck and often do not have any neurological deficit. The size can range from a few millimeters 
to centimeters in diameter. The diagnostic work-up includes the presence of other neurofibromas and any possibility of NF1 and NF2.

Grossly, these tumors are smooth in contour and firm to rubbery hard in consistency. Histologically, tumor cells are fusiform and elongated with the waveform nuclei set in abundant matrix of collagen fibers and mast cells. Tumor is normally smooth surface and slow growing. Specific diagnosis can be made with immunohistochemistry staining for S-100 of neural origin since the S-100 protein is present in all neural tumors. ${ }^{4}$ There are no distinguishing features in neurofibromas that aid in its diagnosis based on clinical grounds alone. Fine needle aspiration cytology, ultrasonography, computed tomography, and magnetic resonance imaging are diagnostic tools for neurofibromas. Differentials include malignant tumors, inflammatory or cystic lesions, lipoma, schwannomas, leiomyomas, granular cell tumors, neuromas, and adenomas. Surgical excision is the treatment of choice with excellent prognosis. Cosmetic outcomes can be prioritized as the tumor is slow growing and noninvasive though complete excision should be ensured to avoid recurrences. The tumors are resistant to radiotherapy and chemotherapy; therefore, these are not indicated.

There have been no reports of neurofibroma involving the subcutaneous tissues of both the cheek and the dorsum of nose as was in our case. There have been isolated reports of neurofibromas affecting oral cavity by Péron et $a l,{ }^{5}$ neurofibromas affecting the maxillary sinus by Nao et al, ${ }^{6}$ oral plexiform neurofibromas by Marocchio et al, ${ }^{2}$ and neurofibroma of lower lip by Mazzoleni et al. ${ }^{7}$ There has been an isolated report of neurofibromatosis of the infraorbital nerve with the involvement of cheek, orbit, and maxillary sinus by Daĭniak et al. ${ }^{8}$

There are reports of endoscopic excision of neurogenic tumors by Kim et al, ${ }^{9}$ and nasal dorsum neurofibroma excision by external rhinoplasty approach by Lee et al. ${ }^{10}$

In this case, as the tumor was restricted to the subcutaneous tissue and there was no intraoral or intranasal extension, it necessitated an external approach to ensure complete excision of the disease.

\section{CONCLUSION}

We report a case of neurofibroma of the subcutaneous tissue of the right cheek and dorsum of nose. Tumor excision was done with local and forehead flap reconstruction. One-year postoperative follow-up reported no recurrence.

\section{REFERENCES}

1. Weber AL, Montandon C, Robson CD. Neurogenic tumors of the neck. Radiol Clin North Am 2000 Sep;38(5):1077-1090.

2. Marocchio LS, Oliveira DT, Pereira MC, Soares CT, Fleury RN. Sporadic and multiple neurofibromas in the head and neck region: a retrospective study of 33 years. Clin Oral Investig 2007 Jun;11(2):165-169.

3. Liapis H, Dehner LP, Gutmann DH. Neurofibroma and cellular neurofibroma with atypia: a report of 14 tumors. Am J Surg Pathol 1999 Sep;23(9):1156-1158.

4. Weiss SW. Neural tumors. In: Weiss SW, editor. Histological typing of soft tissue tumors. New York (NY): Springer, Berlin Heidelberg; 1994. p. 38-39.

5. Péron JM, Auriol M, Ragot JP, Bertrand JC, Szpirglas H, Chomette G, Guilbert F. Neural tumors of the tongue. An anatomicoclinical study apropos of 13 cases. Rev Stomatol Chir Maxillofac 1986;87(5):327-332.

6. Nao EE, Tall A, Ndiaye M, Ndiaye C, Diouf MS, Thiam A, Djafarou AB, Diallo BK, Ndiaye IC, Diouf R, et al. Solitary neurofibroma of the maxillary sinus. Ann Otolaryngol Chir Cervicofac 2009 Nov-Dec;126(5-6):256-258.

7. Mazzoleni G, Civelli M, Paganuzzi F, Priore L. Case report of neurofibroma of the lower lip and left cheek. Riv Ital Stomatol 1982;51(11):945-952.

8. Daĭniak LB, Zagorianskaia ME. Isolated neurofibromatosis of the infraorbital nerve with diffusion into the orbit, maxillary sinus and soft tissues of the cheek. Vestn Otorinolaringol 1965 Sep-Oct;27(5):111-112.

9. Kim YD, Bai CH, Suh JS, Song KW. Transnasal endoscopic excision of an isolated neurofibroma of the nasal septum. Rhinology 1997 Jun;35(2):89-91.

10. Lee JH, Bae JH, Kim KS. A case of solitary neurofibroma of the nasal dorsum: resection using an external rhinoplasty approach. Eur Arch Otorhinolaryngol 2005 Oct;262(10): 813-815. 